\title{
Oscillation criteria of third-order neutral differential equations with damping and distributed deviating arguments
}

Yakun Wang ${ }^{1}$, Fanwei Meng ${ }^{1 *}$ (D) and Juan $\mathrm{Gu}^{2}$

${ }^{*}$ Correspondence:

fwmeng@qfnu.edu.cn

'School of Mathematical Sciences,

Qufu Normal University, Shandong,

P.R. China

Full list of author information is

available at the end of the article

\section{(2) Springer}

\begin{abstract}
Our objective in this paper is to study the oscillatory and asymptotic behavior of the solutions of third-order neutral differential equations with damping and distributed deviating arguments. New oscillation criteria are established, which are based on a refinement generalized Riccati transformation. An important tool for this investigation is the integral averaging technique. Moreover, we provide an example to illustrate the main results.
\end{abstract}

Keywords: Third-order; Neutral differential equations; Oscillation; Riccati transformation

\section{Introduction}

We consider a third-order half-linear neutral differential equation with damping and distributed deviating arguments of the form

$$
\begin{gathered}
\left(\alpha_{2}(\xi)\left(\alpha_{1}(\xi)\left(y^{\prime}(\xi)\right)^{\gamma}\right)^{\prime}\right)^{\prime}+\alpha_{3}(\xi)\left(\alpha_{1}(\xi)\left(y^{\prime}(\xi)\right)^{\gamma}\right)^{\prime} \\
+\int_{c}^{d} F(\xi, s, x(g(\xi, s))) d \eta(s)=0
\end{gathered}
$$

for $\xi>\xi_{0}$, where $y(\xi)=x(\xi)+\int_{a}^{b} p(\xi, \sigma) x(\tau(\xi, \sigma)) d \sigma, \gamma$ is a quotient of odd positive integers.

Throughout the manuscript, we assume the following conditions hold.

$\left(A_{1}\right) \xi_{0}>0$ is a constant, $R=(-\infty, \infty), R^{+}=(0, \infty) . \alpha_{1}(\xi) \in C^{2}\left(\left[\xi_{0}, \infty\right), R^{+}\right)$, $\alpha_{2}(\xi) \in C^{1}\left(\left[\xi_{0}, \infty\right), R^{+}\right), \quad \alpha_{3}(\xi) \in C\left(\left[\xi_{0}, \infty\right), R^{+}\right), \quad \int_{\xi_{0}}^{\infty} \alpha_{1}(\xi)^{-\frac{1}{\gamma}} d \xi=\infty$, $\int_{\xi_{0}}^{\infty} \frac{1}{\alpha_{2}(\xi)} \exp \left(-\int_{\xi_{0}}^{\xi} \frac{\alpha_{3}(s)}{\alpha_{2}(s)} d s\right) d \xi=\infty$.

$\left(A_{2}\right) \tau(\xi, \sigma) \in C^{1}\left(\left[\xi_{0}, \infty\right) \times[a, b], R^{+}\right), \frac{\partial \tau(\xi, \sigma)}{\partial \sigma}>0 . \tau(\xi, \sigma)$ satisfies $\tau(\xi, \sigma) \leq \xi$ and $\lim _{\xi \rightarrow \infty} \tau(\xi, \sigma)=\infty$ for $\sigma \in[a, b], \xi \geq \xi_{0}$. Moreover $p(\xi, \sigma) \in C\left(\left[\xi_{0}, \infty\right) \times[a, b], R^{+}\right)$, $0<p^{*}<1$ is a constant such that $0 \leq P(\xi) \equiv \int_{a}^{b} p(\xi, \sigma) d \sigma<p^{*}$.

$\left(A_{3}\right) F(\xi, s, x) \in C\left(\left[\xi_{0}, \infty\right) \times[c, d] \times(0, \infty), R^{+}\right), q(\xi, s) \in C\left(\left[\xi_{0}, \infty\right) \times[c, d], R^{+}\right), \frac{F(\xi, s, x)}{x^{\gamma}} \geq$ $q(\xi, s)$.

(c) The Author(s) 2021. This article is licensed under a Creative Commons Attribution 4.0 International License, which permits use, sharing, adaptation, distribution and reproduction in any medium or format, as long as you give appropriate credit to the original author(s) and the source, provide a link to the Creative Commons licence, and indicate if changes were made. The images or other third party material in this article are included in the article's Creative Commons licence, unless indicated otherwise in a credit line to the material. If material is not included in the article's Creative Commons licence and your intended use is not permitted by statutory regulation or exceeds the permitted use, you will need to obtain permission directly from the copyright holder. To view a copy of this licence, visit http://creativecommons.org/licenses/by/4.0/. 
$\left(A_{4}\right) g(\xi, s) \in C^{1}\left(\left[\xi_{0}, \infty\right) \times[c, d], R^{+}\right), \frac{\partial g(\xi, s)}{\partial \xi}>0$, and $\frac{\partial g(\xi, s)}{\partial s} \geq 0 . g(\xi, s)$ satisfies $g(\xi, s) \leq \xi$ and $\lim _{\xi \rightarrow \infty} g(\xi, s)=\infty$ for $s \in[c, d], \xi \geq \xi_{0}$.

$\left(A_{5}\right) \eta(s) \in C([c, d], R)$ is strictly increasing and the integral of Eq. (1) is in the sense of Riemann-Stieltjes.

We restrict our attention to those solutions $x(\xi)$ of Eq. (1) which mean $x \in C^{3}\left[L_{x}, \infty\right)$, $L_{x} \geq \xi_{0}$. And Eq. (1) have the property $\alpha_{2}(\xi)\left(\alpha_{1}(\xi)\left(y^{\prime}(\xi)\right)^{\gamma}\right)^{\prime} \in C^{1}\left[L_{x}, \infty\right)$ and $\alpha_{1}(\xi)\left(y^{\prime}(\xi)\right)^{\gamma} \in$ $C^{2}\left[L_{x}, \infty\right)$. We consider only solutions $x$ of the equation which satisfy $\sup \{|x(\xi)|: \xi \geq L\}>$ 0 for all $L>L_{x}$. As usual, a nontrivial solution of Eq. (1)is called oscillatory if it has arbitrarily large zero; otherwise, it is said to be nonoscillatory. Equation (1) is called oscillatory if all of its solutions are oscillatory.

As a modeling tool for many phenomena in different fields, differential equations are difficult to provide general solutions for most of them. Therefore, the qualitative properties of differential equations have attracted a lot of attention from scholars. Oscillation and asymptotic property are important parts of qualitative research, and they are still hot topics. In the process of studying the problem, it is not difficult to find that the future state is not only affected by the current state but also by the past state. Therefore, adding some time delay into the equation is more beneficial to the description of the problem. In addition to the theoretical importance, the qualitative study of neutral equations has great practical importance. Interested readers can read Chap. 11 in the book [1] and book [2]. In particular, half-linear equations arise in the study of $p$-Laplace equations, non-Newtonian fluid theory, porous medium problems, chemotaxis models, and so on; see, e.g., the papers [3-8] for more details.

With the progress and development of science and technology, in recent decades, oscillation results of third-order neutral delay (TOND) differential equations have attracted extensive attention. In 2019, Wei et al. [9] and [10] studied the TOND differential equations with distributed deviating arguments and damping

$$
\left(\alpha_{2}(\xi)\left(\alpha_{1}(\xi) y^{\prime}(\xi)\right)^{\prime}\right)^{\prime}+\alpha_{3}(\xi)\left(\alpha_{1}(\xi) y^{\prime}(\xi)\right)^{\prime}+\int_{c}^{d} F(\xi, s, x(g(\xi, s))) d \eta(s)=0
$$

And new canonical conditions are given as

$$
\int_{\xi_{0}}^{\infty} \alpha_{1}(\xi)^{-\frac{1}{\gamma}} d \xi=\infty \text { and } \int_{\xi_{0}}^{\infty} \frac{1}{\alpha_{2}(\xi)} \exp \left(-\int_{\xi_{0}}^{\xi} \frac{\alpha_{3}(s)}{\alpha_{2}(s)} d s\right) d \xi=\infty
$$

Under this condition, a new method to deal with damping terms is given by constructing an exponential function. However, in the study of many TOND differential equations or dynamic equations, half-linear equations are still of great significance. Inspired by the articles [11-13], we consider the form of Eq. (1) based on Eq. (2). We point out that one of the key issues for the study is to find canonical conditions and an asymptotic condition similar to the study of Eq. (2). In order to solve this problem, we refer to literature [14-16] and give the conditions corresponding of Eq. (2) in this paper.

In addition, there is a large number of papers showing that the research on the oscillation of the third-order delay differential equation is related to the oscillation of the secondorder delay differential equation. We direct interested readers to [12]. A natural question is whether Eq. (1) can apply the second-order method. Thus, to enrich the method of halflinear TOND differential equations with damping, here, we introduce several interesting 
approaches to second-order differential equations oscillation problems. In [17-20], a new operator is created. The operator is flexible in application and it is superior in estimation parameters of some second-order equations. In [21] and [22], an unusual method is also presented. That is, the new oscillation theorem is obtained by taking the inverse of the Riccati function. Therefore, another difficulty is how to effectively generalize these methods to Eq. (1) and get some new results. And in particular, note that all of the results in this paper apply to Eq. (2) when we set $\gamma=1$.

Next, we give the Definition 1 we need to use.

Definition 1 ([17]) We say that a function $\Phi=\Phi(\xi, h, l)$ belongs to the function class $Y$, denoted by $\Phi \in Y$, if $\Phi \in C(E, R)$, where $E=\left\{(\xi, h, l): \xi_{0} \leq l \leq h \leq \xi<\infty\right\}$, which satisfies $\Phi(\xi, \xi, l)=0, \Phi(\xi, l, l)=0, \Phi(\xi, h, l) \neq 0$ for $l<h<\xi$, and has the partial derivative $\frac{\partial \Phi}{\partial h}$ on $E$ such that $\frac{\partial \Phi}{\partial h}$ is locally integrable with respect to $h \in E$. We define the operator $A[\cdot ; l, \xi]$ by

$$
A[\psi ; l, \xi]=\int_{l}^{\xi} \Phi^{2}(\xi, h, l) \psi(h) d h
$$

for $\xi \geq h \geq l \geq \xi_{0}$ and $\psi(h) \in C^{1}\left(\left[\xi_{0}, \infty\right), R\right)$. The function $\varphi=\varphi(\xi, h, l)$ is defined by

$$
\frac{\partial \Phi(\xi, h, l)}{\partial h}=\varphi(\xi, h, l) \Phi(\xi, h, l) .
$$

It is easy to verify that $A[\cdot ; l, \xi]$ is a linear operator and that it satisfies

$$
A\left[\psi^{\prime} ; l, \xi\right]=-2 A[\psi \varphi ; l, \xi]
$$

for $\psi(h) \in C^{1}\left(\left[\xi_{0}, \infty\right), R\right)$.

\section{Preliminaries}

As we all know, the common method to discuss the oscillation property of the solution is to assume that the equation has an eventually positive solution. In this part, we study and classify the function $y(\xi)$ related to the eventually positive solution. And during the following sections of our paper, we shall need the next lemmas.

Lemma 2.1 ([23]) Set $B(v)=b_{1}(\xi) v-b_{2}(\xi) v^{\frac{\gamma+1}{\gamma}}$, where the function $b_{1}(\xi)$ is arbitrary, function $b_{2}(\xi)$ and function $v$ are always positive, $\gamma$ is a positive constant. Then the function $B(v)$ has the maximum value $B_{\max }$ at $v_{0}$ on $R$ such that

$$
B(v) \leq B_{\max }=\frac{\gamma^{\gamma}}{(\gamma+1)^{\gamma+1}} \frac{b_{1}^{\gamma+1}(\xi)}{b_{2}^{\gamma}(\xi)}
$$

where $v_{0}=\left(\frac{\gamma}{\gamma+1} \frac{b_{1}(\xi)}{b_{2}(\xi)}\right)^{\gamma}$.

Lemma 2.2 Suppose that $\left(A_{1}\right),\left(A_{3}\right)$, and $\left(A_{5}\right)$ hold. Let $x(\xi)$ be a positive solution of Eq. (1). Then $y(\xi)=x(\xi)+\int_{a}^{b} p(\xi, \sigma) x(\tau(\xi, \sigma)) d \sigma$ must be in one of the following two cases:

$$
\begin{aligned}
& \text { case 1: } \quad y(\xi)>0, \quad y^{\prime}(\xi)>0, \quad\left(\alpha_{1}(\xi)\left(y^{\prime}(\xi)\right)^{\gamma}\right)^{\prime}>0 ; \\
& \text { case } 2: \quad y(\xi)>0, \quad y^{\prime}(\xi)<0, \quad\left(\alpha_{1}(\xi)\left(y^{\prime}(\xi)\right)^{\gamma}\right)^{\prime}>0 \text {, }
\end{aligned}
$$

where $\xi \geq \xi_{1} \geq \xi_{0}$ for sufficiently large constant $\xi_{1}$. 
Proof Suppose that $x(\xi)$ is the positive solution of Eq. (1) in $\left[\xi_{0}, \infty\right)$. There exists sufficiently large $\xi_{1}>\xi_{0}$ such that $x(\tau(\xi, \sigma))>0$ and $x(g(\xi, s))>0$ for $\xi>\xi_{1}$. From $y(\xi)=$ $x(\xi)+\int_{a}^{b} p(\xi, \sigma) x(\tau(\xi, \sigma)) d \sigma$, we get $y(\xi)>x(\xi)>0$.

Based on Eq. (1), $\left(A_{3}\right)$, and $\left(A_{5}\right)$, we obtain

$$
\begin{aligned}
& \left(\alpha_{2}(\xi)\left(\alpha_{1}(\xi)\left(y^{\prime}(\xi)\right)^{\gamma}\right)^{\prime}\right)^{\prime}+\alpha_{3}(\xi)\left(\alpha_{1}(\xi)\left(y^{\prime}(\xi)\right)^{\gamma}\right)^{\prime} \\
& \quad=-\int_{c}^{d} F(\xi, s, x(g(\xi, s))) d \eta(s) \\
& \quad \leq-\int_{c}^{d} q(\xi, s) x^{\gamma}(g(\xi, s)) d \eta(s)<0 .
\end{aligned}
$$

To combine the damping terms, we use a useful function $z(\xi)=\exp \left(\int_{\xi_{0}}^{\xi} \frac{\alpha_{3}(s)}{\alpha_{2}(s)} d s\right)$. By the properties of the exponential function, we have $z(\xi)>0$. Multiplying both sides of inequality (7) with $z(\xi)$, we get

$$
\begin{aligned}
& z(\xi)\left(\left(\alpha_{2}(\xi)\left(\alpha_{1}(\xi)\left(y^{\prime}(\xi)\right)^{\gamma}\right)^{\prime}\right)^{\prime}+\alpha_{3}(\xi)\left(\alpha_{1}(\xi)\left(y^{\prime}(\xi)\right)^{\gamma}\right)^{\prime}\right) \\
& =\left(z(\xi) \alpha_{2}(\xi)\left(\alpha_{1}(\xi)\left(y^{\prime}(\xi)\right)^{\gamma}\right)^{\prime}\right)^{\prime}<0,
\end{aligned}
$$

which means that $z(\xi) \alpha_{2}(\xi)\left(\alpha_{1}(\xi)\left(y^{\prime}(\xi)\right)^{\gamma}\right)^{\prime}$ is decreasing and eventually of one sign. Due to $z(\xi)>0$ and $\alpha_{2}(\xi)>0$, we obtain

$$
\left(\alpha_{1}(\xi)\left(y^{\prime}(\xi)\right)^{\gamma}\right)^{\prime}>0 \quad \text { or } \quad\left(\alpha_{1}(\xi)\left(y^{\prime}(\xi)\right)^{\gamma}\right)^{\prime}<0
$$

for $\xi>\xi_{1}$. Then we assert that $\left(\alpha_{1}(\xi)\left(y^{\prime}(\xi)\right)^{\gamma}\right)^{\prime}>0$. If $\left(\alpha_{1}(\xi)\left(y^{\prime}(\xi)\right)^{\gamma}\right)^{\prime}<0$, by $(8)$, there exists $\xi_{2}>\xi_{1}$, when $\xi>\xi_{2}$, we have

$$
z(\xi) \alpha_{2}(\xi)\left(\alpha_{1}(\xi)\left(y^{\prime}(\xi)\right)^{\gamma}\right)^{\prime}<z\left(\xi_{2}\right) \alpha_{2}\left(\xi_{2}\right)\left(\alpha_{1}\left(\xi_{2}\right)\left(y^{\prime}\left(\xi_{2}\right)\right)^{\gamma}\right)^{\prime}=:-M_{1},
$$

where $M_{1}$ is a positive constant. Integrating (9) from $\xi_{2}$ to $\xi$, we have

$$
\begin{aligned}
\alpha_{1}(\xi)\left(y^{\prime}(\xi)\right)^{\gamma} & <\alpha_{1}\left(\xi_{2}\right)\left(y^{\prime}\left(\xi_{2}\right)\right)^{\gamma}-\int_{\xi_{2}}^{\xi} \frac{M_{1}}{z(\varsigma) \alpha_{2}(\varsigma)} d \varsigma \\
& =\alpha_{1}\left(\xi_{2}\right)\left(y^{\prime}\left(\xi_{2}\right)\right)^{\gamma}-M_{1} \int_{\xi_{2}}^{\xi} \frac{1}{\alpha_{2}(\varsigma)} \exp \left(-\int_{\xi_{0}}^{\varsigma} \frac{\alpha_{3}(s)}{\alpha_{2}(s)} d s\right) d \varsigma .
\end{aligned}
$$

From $\left(A_{1}\right)$, we get $\alpha_{1}(\xi)\left(y^{\prime}(\xi)\right)^{\gamma}<0$, as $\xi \rightarrow \infty$, which means $y^{\prime}(\xi)<0$. Due to $\left(\alpha_{1}(\xi)\left(y^{\prime}(\xi)\right)^{\gamma}\right)^{\prime}<0$, there exists $\xi_{3}>\xi_{2}$, when $\xi>\xi_{3}$, we have

$$
\alpha_{1}(\xi)\left(y^{\prime}(\xi)\right)^{\gamma}<\alpha_{1}\left(\xi_{3}\right)\left(y^{\prime}\left(\xi_{3}\right)\right)^{\gamma}=:-M_{2},
$$

where $M_{2}$ is a positive constant. Then, integrating (11) from $\xi_{3}$ to $\xi$, we have

$$
y(\xi)<y\left(\xi_{3}\right)-M_{2}^{\frac{1}{\gamma}} \int_{\xi_{3}}^{\xi} \alpha_{1}(s)^{-\frac{1}{\gamma}} d s .
$$

From $\left(A_{1}\right)$, we can get $y(\xi)<0$, as $\xi \rightarrow \infty$, which contradicts $y(\xi)>x(\xi)>0$. Thus, $\left(\alpha_{1}(\xi)\left(y^{\prime}(\xi)\right)^{\gamma}\right)^{\prime}>0$. 
Next, we prove that $y^{\prime}(\xi)$ is eventually of one sign. By $\left(\alpha_{1}(\xi)\left(y^{\prime}(\xi)\right)^{\gamma}\right)^{\prime}>0$, we have that $\alpha_{1}(\xi)\left(y^{\prime}(\xi)\right)^{\gamma}$ is increasing and eventually of one sign. From $\alpha_{1}(\xi)>0$ and $\gamma$ is a quotient of odd positive integers, we get that $y^{\prime}(\xi)$ is eventually of one sign. So, it is easy to know that $y(\xi)$ has case 1 or case 2 . This completes the proof.

Lemma 2.3 Suppose that $\left(A_{1}\right)-\left(A_{5}\right)$ hold. Let $x(\xi)$ be a positive solution of Eq. (1), and $y(\xi)$ satisfies case 2 in Lemma 2.2. If

$$
\int_{\xi_{0}}^{\infty}\left(\frac{1}{\alpha_{1}(u)} \int_{u}^{\infty} \frac{1}{\alpha_{2}(v)} \int_{v}^{\infty} q(s) d s d v\right)^{\frac{1}{\gamma}} d u=\infty
$$

where $q(\xi)=\int_{c}^{d} q(\xi, s) d \eta(s)$ for $\xi \in\left(\xi_{0}, \infty\right)$, then $\lim _{\xi \rightarrow \infty} x(\xi)=0$.

Proof Suppose that $x(\xi)$ is the positive solution of Eq. (1) in $\left[\xi_{0}, \infty\right)$. From Lemma 2.2, we have $y(\xi)>0, y^{\prime}(\xi)<0$. Based on the simple analysis, function $y(\xi)$ must converge to the nonnegative constant $m$, when $\xi \rightarrow \infty$. And we assert $\lim _{\xi \rightarrow \infty} y(\xi)=m=0$. If $m>0$, noting that $y(\xi)$ is decreasing for $\xi \geq \xi_{1} \geq \xi_{0}$, hence, we always have a constant $\theta>0$ such that $m<y(\xi)<m+\theta$. From $\left(A_{2}\right)$, we have a constant $0<p^{*}<1$, because of the Archimedes property of real numbers, we can choose a special number $\theta$ such that $0<\theta<\frac{m\left(1-p^{*}\right)}{p^{*}}$.

Next, from $\left(A_{2}\right)$ and integral mean value theorem, use $m<y(\xi)<m+\theta$, we have

$$
\begin{aligned}
x(\xi) & =y(\xi)-\int_{a}^{b} p(\xi, \sigma) x(\tau(\xi, \sigma)) d \sigma \\
& \geq y(\xi)-\int_{a}^{b} p(\xi, \sigma) y(\tau(\xi, \sigma)) d \sigma \\
& \geq y(\xi)-y(\tau(\xi, a)) \int_{a}^{b} p(\xi, \sigma) d \sigma \\
& \geq m-p^{*}(m+\theta) \geq \frac{m-p^{*}(m+\theta)}{m+\theta}(m+\theta) \\
& \geq \frac{m-p^{*}(m+\theta)}{m+\theta} y(\xi) .
\end{aligned}
$$

From $0<\theta<\frac{m\left(1-p^{*}\right)}{p^{*}}$, we obtain $\frac{m-p^{*}(m+\theta)}{m+\theta}>0$. Then, using Eq. (1), we have

$$
\begin{aligned}
& \left(\alpha_{2}(\xi)\left(\alpha_{1}(\xi)\left(y^{\prime}(\xi)\right)^{\gamma}\right)^{\prime}\right)^{\prime}+\alpha_{3}(\xi)\left(\alpha_{1}(\xi)\left(y^{\prime}(\xi)\right)^{\gamma}\right)^{\prime} \\
& \quad=-\int_{c}^{d} F(\xi, s, x(g(\xi, s))) d \eta(s) \leq-\int_{c}^{d} q(\xi, s) x^{\gamma}(g(\xi, s)) d \eta(s) \\
& \leq-\int_{c}^{d} q(\xi, s)\left(\frac{m-p^{*}(m+\theta)}{m+\theta} y(g(\xi, s))\right)^{\gamma} d \eta(s) \\
& \leq-\left(\frac{m-p^{*}(m+\theta)}{m+\theta}\right)^{\gamma} y^{\gamma}(g(\xi, d)) q(\xi) \\
& \leq-\left(\frac{m-p^{*}(m+\theta)}{m+\theta}\right)^{\gamma} y^{\gamma}(\xi) q(\xi) .
\end{aligned}
$$


We also use function $z(\xi)=\exp \left(\int_{\xi_{0}}^{\xi} \frac{\alpha_{3}(s)}{\alpha_{2}(s)} d s\right)>0$, and we have $z^{\prime}(\xi)>0$ easily. Then

$$
\begin{aligned}
& \left(z(\xi) \alpha_{2}(\xi)\left(\alpha_{1}(\xi)\left(y^{\prime}(\xi)\right)^{\gamma}\right)^{\prime}\right)^{\prime} \\
& \quad=z(\xi)\left(\left(\alpha_{2}(\xi)\left(\alpha_{1}(\xi)\left(y^{\prime}(\xi)\right)^{\gamma}\right)^{\prime}\right)^{\prime}+\alpha_{3}(\xi)\left(\alpha_{1}(\xi)\left(y^{\prime}(\xi)\right)^{\gamma}\right)^{\prime}\right) \\
& \quad \leq-\left(\frac{m-p^{*}(m+\theta)}{m+\theta}\right)^{\gamma} y^{\gamma}(\xi) z(\xi) q(\xi) .
\end{aligned}
$$

Integrating (16) from $\xi$ to $\infty$, using $m<y(\xi)<m+\theta$, we get

$$
\begin{aligned}
-z(\xi) \alpha_{2}(\xi)\left(\alpha_{1}(\xi)\left(y^{\prime}(\xi)\right)^{\gamma}\right)^{\prime} & <-\left(\frac{m-p^{*}(m+\theta)}{m+\theta}\right)^{\gamma} \int_{\xi}^{\infty} y^{\gamma}(s) z(s) q(s) d s \\
& <-\left(\frac{m\left(m-p^{*}(m+\theta)\right)}{m+\theta}\right)^{\gamma} z(\xi) \int_{\xi}^{\infty} q(s) d s,
\end{aligned}
$$

which implies that

$$
\left(\alpha_{1}(\xi)\left(y^{\prime}(\xi)\right)^{\gamma}\right)^{\prime}>\left(\frac{m\left(m-p^{*}(m+\theta)\right)}{m+\theta}\right)^{\gamma} \frac{1}{\alpha_{2}(\xi)} \int_{\xi}^{\infty} q(s) d s .
$$

Integrating (18) from $\xi$ to $\infty$, we have

$$
\alpha_{1}(\xi)\left(y^{\prime}(\xi)\right)^{\gamma}<-\left(\frac{m\left(m-p^{*}(m+\theta)\right)}{m+\theta}\right)^{\gamma} \int_{\xi}^{\infty} \frac{1}{\alpha_{2}(v)} \int_{v}^{\infty} q(s) d s d v
$$

Integrating (19) from $\xi_{1}$ to $\infty$, we get

$$
\int_{\xi_{1}}^{\infty}\left(\frac{1}{\alpha_{1}(u)} \int_{u}^{\infty} \frac{1}{\alpha_{2}(v)} \int_{v}^{\infty} q(s) d s d v\right)^{\frac{1}{\gamma}} d u<\frac{m+\theta}{m\left(m-p^{*}(m+\theta)\right)} y\left(\xi_{1}\right)
$$

This contradicts (13), and we get $m=0$. Due to $\lim _{\xi \rightarrow \infty} y(\xi)=0$ and $y(\xi)>x(\xi)>0$, we obtain $\lim _{\xi \rightarrow \infty} x(\xi)=0$. This completes the proof.

\section{Main results}

In this section, we establish some new oscillation criteria for Eq. (1). For convenience, we denote

$$
\begin{aligned}
& q(\xi)=\int_{c}^{d} q(\xi, s) d \eta(s), \quad g^{\prime}(\xi, c)=\frac{\partial g(\xi, c)}{\partial \xi}, \quad \rho^{\prime}(\xi, h, l)=\frac{\partial \rho(\xi, h, l)}{\partial h} \\
& G(\xi):=g^{\prime}(\xi, c)\left(\frac{1}{\alpha_{1}(g(\xi, c))}\right)^{\frac{1}{\gamma}}\left(\int_{\xi_{1}}^{g(\xi, c)} \frac{1}{\alpha_{2}(s)} d s\right)^{\frac{1}{\gamma}}, \\
& R(\xi, h, l)=\frac{\rho^{\prime}(\xi, h, l)}{\rho(\xi, h, l)}-\frac{\alpha_{3}(\xi)}{\alpha_{2}(\xi)}+2 \varphi(\xi, h, l) .
\end{aligned}
$$

Theorem 3.1 Let $\left(A_{1}\right)-\left(A_{5}\right)$ and (13) hold. Assume that, for each $l \geq \xi_{0}$, there exist a function $\Phi \in Y$ and a positive function $\rho \in C^{1}\left(\left[\xi_{0}, \infty\right), R^{+}\right)$such that

$$
\limsup _{\xi \rightarrow \infty} A\left[\rho\left(1-p^{*}\right)^{\gamma} q-\left(\frac{\frac{\rho^{\prime}}{\rho}-\frac{\alpha_{3}}{\alpha_{2}}+2 \varphi}{\gamma+1}\right)^{\gamma+1} \frac{\rho}{G^{\gamma}} ; l, \xi\right]>0
$$


where the operator $A$ is defined by (3), and $\varphi=\varphi(\xi, h, l)$ is defined by (4). Then every solution of Eq. (1) is oscillatory or converges to zero.

Proof Suppose to the contrary that there exists an eventually positive solution $x(\xi)$ of Eq. (1) such that $x(\xi)>0, x(\tau(\xi, \sigma))>0, x(g(\xi, s))>0$ for $\xi>\xi_{1}>\xi_{0}$. Then $y(\xi)=x(\xi)+$ $\int_{a}^{b} p(\xi, \sigma) x(\tau(\xi, \sigma)) d \sigma>0$ for $\xi>\xi_{1}>\xi_{0}$. From Lemma 2.2, we get that $y(\xi)$ is one of case 1 or case 2 .

If $y(\xi)$ satisfies case 1 , we can get

$$
\begin{aligned}
x(\xi) & =y(\xi)-\int_{a}^{b} p(\xi, \sigma) x(\tau(\xi, \sigma)) d \sigma \\
& \geq y(\xi)-\int_{a}^{b} p(\xi, \sigma) y(\tau(\xi, \sigma)) d \sigma \\
& \geq y(\xi)-y(\xi) \int_{a}^{b} p(\xi, \sigma) d \sigma \\
& \geq\left(1-p^{*}\right) y(\xi) .
\end{aligned}
$$

Combining (1) and (22), we have

$$
\begin{aligned}
& \left(\alpha_{2}(\xi)\left(\alpha_{1}(\xi)\left(y^{\prime}(\xi)\right)^{\gamma}\right)^{\prime}\right)^{\prime}+\alpha_{3}(\xi)\left(\alpha_{1}(\xi)\left(y^{\prime}(\xi)\right)^{\gamma}\right)^{\prime} \\
& \quad=-\int_{c}^{d} F(\xi, s, x(g(\xi, s))) d \eta(s) \\
& \quad \leq-\int_{c}^{d} q(\xi, s) x^{\gamma}(g(\xi, s)) d \eta(s) \\
& \quad \leq-\left(1-p^{*}\right)^{\gamma} y^{\gamma}(g(\xi, c)) q(\xi) .
\end{aligned}
$$

Since $y(\xi)$ has case 1 , we get $y^{\prime}(\xi)>0$ and $\left(\alpha_{1}(\xi)\left(y^{\prime}(\xi)\right)^{\gamma}\right)^{\prime}>0$. From (23), we can easily get $\left(\alpha_{2}(\xi)\left(\alpha_{1}(\xi)\left(y^{\prime}(\xi)\right)^{\gamma}\right)^{\prime}\right)^{\prime}<0$, and we have

$$
\begin{aligned}
\alpha_{1}(\xi)\left(y^{\prime}(\xi)\right)^{\gamma} & =\alpha_{1}\left(\xi_{1}\right)\left(y^{\prime}\left(\xi_{1}\right)\right)^{\gamma}+\int_{\xi_{1}}^{\xi}\left(\alpha_{1}(s)\left(y^{\prime}(s)\right)^{\gamma}\right)^{\prime} d s \\
& \geq \int_{\xi_{1}}^{\xi}\left(\alpha_{1}(s)\left(y^{\prime}(s)\right)^{\gamma}\right)^{\prime} d s \\
& \geq \int_{\xi_{1}}^{\xi} \frac{\alpha_{2}(s)\left(\alpha_{1}(s)\left(y^{\prime}(s)\right)^{\gamma}\right)^{\prime}}{\alpha_{2}(s)} d s \\
& \geq \alpha_{2}(\xi)\left(\alpha_{1}(\xi)\left(y^{\prime}(\xi)\right)^{\gamma}\right)^{\prime} \int_{\xi_{1}}^{\xi} \frac{1}{\alpha_{2}(s)} d s .
\end{aligned}
$$

That means

$$
y^{\prime}(\xi) \geq\left(\frac{\alpha_{2}(\xi)\left(\alpha_{1}(\xi)\left(y^{\prime}(\xi)\right)^{\gamma}\right)^{\prime}}{\alpha_{1}(\xi)}\right)^{\frac{1}{\gamma}}\left(\int_{\xi_{1}}^{\xi} \frac{1}{\alpha_{2}(s)} d s\right)^{\frac{1}{\gamma}} .
$$


From $\left(A_{4}\right)$, we obtain

$$
\begin{aligned}
& y^{\prime}(g(\xi, c)) \\
& \quad \geq\left(\frac{\alpha_{2}(g(\xi, c))\left(\alpha_{1}(g(\xi, c))\left(y^{\prime}(g(\xi, c))\right)^{\gamma}\right)^{\prime}}{\alpha_{1}(g(\xi, c))}\right)^{\frac{1}{\gamma}}\left(\int_{\xi_{1}}^{g(\xi, c)} \frac{1}{\alpha_{2}(s)} d s\right)^{\frac{1}{\gamma}} \\
& \left.\quad \geq\left(\frac{\alpha_{2}(\xi)\left(\alpha_{1}(\xi)\left(y^{\prime}(\xi)\right)^{\gamma}\right)^{\prime}}{\alpha_{1}(g(\xi, c))}\right)^{\frac{1}{\gamma}}\left(\int_{\xi_{1}}^{g(\xi, c)} \frac{1}{\alpha_{2}(s)} d s\right)^{\frac{1}{\gamma}}\right)^{\frac{1}{\gamma}}\left(\int_{\xi_{1}}^{g(\xi, c)} \frac{1}{\alpha_{2}(s)} d s\right)^{\frac{1}{\gamma}} .
\end{aligned}
$$

Next, we define the generalized Riccati-type function

$$
w(h)=\rho(h) \frac{\alpha_{2}(h)\left(\alpha_{1}(h)\left(y^{\prime}(h)\right)^{\gamma}\right)^{\prime}}{y^{\gamma}(g(h, c))} \text { for } h \geq \xi_{1} .
$$

Obviously, $w(h)>0$. Differentiating $w(h)$, we obtain

$$
\begin{aligned}
w^{\prime}(h)= & \rho^{\prime}(h) \frac{\alpha_{2}(h)\left(\alpha_{1}(h)\left(y^{\prime}(h)\right)^{\gamma}\right)^{\prime}}{y^{\gamma}(g(h, c))}+\rho(h) \frac{\left(\alpha_{2}(h)\left(\alpha_{1}(h)\left(y^{\prime}(h)\right)^{\gamma}\right)^{\prime}\right)^{\prime}}{y^{\gamma}(g(h, c))} \\
& -\gamma \rho(h) \frac{\alpha_{2}(h)\left(\alpha_{1}(\xi)\left(y^{\prime}(h)\right)^{\gamma}\right)^{\prime} y^{\prime}(g(h, c)) g^{\prime}(h, c)}{y^{\gamma+1}(g(h, c))} .
\end{aligned}
$$

From (23), (24), (26), and (27), by simple computation, we have

$$
\begin{aligned}
w^{\prime}(h) \leq & \frac{\rho^{\prime}(h)}{\rho(h)}\left(\rho(h) \frac{\alpha_{2}(h)\left(\alpha_{1}(h)\left(y^{\prime}(h)\right)^{\gamma}\right)^{\prime}}{y^{\gamma}(g(h, c))}\right) \\
& -\rho(h) \frac{\alpha_{3}(h)\left(\alpha_{1}(h)\left(y^{\prime}(h)\right)^{\gamma}\right)^{\prime}}{y^{\gamma}(g(h, c))} \\
& -\rho(h) \frac{\left(1-p^{*}\right)^{\gamma} y^{\gamma}(g(h, c)) q(h)}{y^{\gamma}(g(h, c))} \\
& -\gamma \rho(h) \frac{\alpha_{2}(h)\left(\alpha_{1}(h)\left(y^{\prime}(h)\right)^{\gamma}\right)^{\prime} y^{\prime}(g(h, c)) g^{\prime}(h, c)}{y^{\gamma+1}(g(h, c))} \\
\leq & \frac{\rho^{\prime}(h)}{\rho(h)} w(h)-\frac{\alpha_{3}(h)}{\alpha_{2}(h)} w(h)-\rho(h)\left(1-p^{*}\right)^{\gamma} q(h) \\
& -\frac{\gamma y^{\prime}(g(h, c)) g^{\prime}(h, c)}{y(g(h, c))} w(h) \\
\leq & \frac{\rho^{\prime}(h)}{\rho(h)} w(h)-\frac{\alpha_{3}(h)}{\alpha_{2}(h)} w(h)-\rho(h)\left(1-p^{*}\right)^{\gamma} q(h) \\
& -\frac{\gamma g^{\prime}(h, c)\left(\frac{1}{\alpha_{1}(g(h, c))}\right)^{\frac{1}{\gamma}}\left(\int_{\xi_{1}}^{g(h, c)} \frac{1}{\alpha_{2}(s)} d s\right)^{\frac{1}{\gamma}}(h)}{w^{\frac{\gamma}{\gamma}}(h) .}
\end{aligned}
$$


For convenience, let $G(h):=g^{\prime}(h, c)\left(\frac{1}{\alpha_{1}(g(h, c))}\right)^{\frac{1}{\gamma}}\left(\int_{\xi_{1}}^{g(h, c)} \frac{1}{\alpha_{2}(s)} d s\right)^{\frac{1}{\gamma}}$. Then we obtain

$$
\begin{aligned}
w^{\prime}(h) \leq & \left(\frac{\rho^{\prime}(h)}{\rho(h)}-\frac{\alpha_{3}(h)}{\alpha_{2}(h)}\right) w(h) \\
& -\gamma \rho^{-\frac{1}{\gamma}}(h) G(h) w^{\frac{\gamma+1}{\gamma}}(h)-\rho(h)\left(1-p^{*}\right)^{\gamma} q(h) .
\end{aligned}
$$

Applying the operator $A\left[\cdot ; \xi_{1}, \xi\right]\left(\xi \geq \xi_{1}\right)$ to $(28)$, we note that $A\left[\cdot ; \xi_{1}, \xi\right]$ is a linear operator, then we get

$$
A\left[w^{\prime} ; \xi_{1}, \xi\right] \leq A\left[\left(\frac{\rho^{\prime}}{\rho}-\frac{\alpha_{3}}{\alpha_{2}}\right) w-\gamma \rho^{-\frac{1}{\gamma}} G w^{\frac{\gamma+1}{\gamma}} ; \xi_{1}, \xi\right]-A\left[\rho\left(1-p^{*}\right)^{\gamma} q ; \xi_{1}, \xi\right]
$$

By (5) and the above inequality, we have

$$
A\left[\rho\left(1-p^{*}\right)^{\gamma} q ; \xi_{1}, \xi\right] \leq A\left[\left(\frac{\rho^{\prime}}{\rho}-\frac{\alpha_{3}}{\alpha_{2}}+2 \varphi\right) w-\gamma \rho^{-\frac{1}{\gamma}} G w^{\frac{\gamma+1}{\gamma}} ; \xi_{1}, \xi\right]
$$

To use Lemma 2.1, set

$$
b_{1}=\frac{\rho^{\prime}}{\rho}-\frac{\alpha_{3}}{\alpha_{2}}+2 \varphi, \quad b_{2}=\gamma \rho^{-\frac{1}{\gamma}} G, \quad v=w .
$$

We can see that

$$
A\left[\rho\left(1-p^{*}\right)^{\gamma} q ; \xi_{1}, \xi\right] \leq A\left[\left(\frac{\frac{\rho^{\prime}}{\rho}-\frac{\alpha_{3}}{\alpha_{2}}+2 \varphi}{\gamma+1}\right)^{\gamma+1} \frac{\rho}{G^{\gamma}} ; \xi_{1}, \xi\right]
$$

That means

$$
A\left[\rho\left(1-p^{*}\right)^{\gamma} q-\left(\frac{\frac{\rho^{\prime}}{\rho}-\frac{\alpha_{3}}{\alpha_{2}}+2 \varphi}{\gamma+1}\right)^{\gamma+1} \frac{\rho}{G^{\gamma}} ; \xi_{1}, \xi\right] \leq 0 .
$$

Taking the super limit in the above inequality, we arrive at this inequality

$$
\limsup _{\xi \rightarrow \infty} A\left[\rho\left(1-p^{*}\right)^{\gamma} q-\left(\frac{\frac{\rho^{\prime}}{\rho}-\frac{\alpha_{3}}{\alpha_{2}}+2 \varphi}{\gamma+1}\right)^{\gamma+1} \frac{\rho}{G^{\gamma}} ; \xi_{1}, \xi\right] \leq 0
$$

which is a contradiction to (21).

If $y(\xi)$ satisfies case 2, from Lemma 2.3, the solution of (1) converges to zero. The proof is complete.

Next, let us discuss the special function $\rho(\xi)$ in Riccati-type function (27), then we can get some new oscillation criteria that are weaker than Theorem 3.1.

Theorem 3.2 Let $\left(A_{1}\right)-\left(A_{5}\right)$ and (13) hold. Assume that, for each $l \geq \xi_{0}$, there exists a function $\Phi \in Y$ such that

$$
\limsup _{\xi \rightarrow \infty} A\left[\rho\left(1-p^{*}\right)^{\gamma} q ; l, \xi\right]>0,
$$


where the operator $A$ is defined by (3), and $\rho=\exp \left(\int_{\xi_{1}}^{h}\left(\frac{\alpha_{3}(s)}{\alpha_{2}(s)}-2 \varphi(\xi, s, l)\right) d s\right)$ for $\varphi(\xi, s, l)$ is as in (4). Then every solution of Eq. (1) is oscillatory or converges to zero.

Proof Suppose the contrary. Let $x(\xi)$ be an eventually positive solution of Eq. (1). Similar to the proof of Theorem 3.1, if we set $\rho=\exp \left(\int_{\xi_{1}}^{h}\left(\frac{\alpha_{3}(s)}{\alpha_{2}(s)}-2 \varphi(\xi, s, l)\right) d s\right)$, then (27) becomes

$$
w(\xi, h, l)=\rho(\xi, h, l) \frac{\alpha_{2}(h)\left(\alpha_{1}(h)\left(y^{\prime}(h)\right)^{\gamma}\right)^{\prime}}{y^{\gamma}(g(h, c))} .
$$

Symbolize $\frac{\partial w(\xi, h, l)}{\partial h}:=w^{\prime}(\xi, h, l)$ and $\frac{\partial \rho(\xi, h, l)}{\partial h}:=\rho^{\prime}(\xi, h, l)$. We can easily get the operator $A[\cdot ; l, \xi]$ satisfying (5) for each fixed $\xi$ and $l$, where $\xi>h>l>\xi_{1}$. Then we also get inequality (30). By simple calculation, we obtain

$$
\begin{aligned}
\rho^{\prime}(\xi, h, l) & =\left(\frac{\alpha_{3}(h)}{\alpha_{2}(h)}-2 \varphi(\xi, h, l)\right) \exp \left(\int_{\xi_{1}}^{h}\left(\frac{\alpha_{3}(s)}{\alpha_{2}(s)}-2 \varphi(\xi, s, l)\right) d s\right) \\
& =\left(\frac{\alpha_{3}(h)}{\alpha_{2}(h)}-2 \varphi(\xi, h, l)\right) \rho(\xi, h, l),
\end{aligned}
$$

and $R(\xi, h, l)=\frac{\rho^{\prime}(\xi, h, l)}{\rho(\xi, h, l)}-\frac{\alpha_{3}(h)}{\alpha_{2}(h)}+2 \varphi(\xi, h, l)=0$ in inequality (30). This means that, for every $\xi$ and $l$, where $\xi>h>l>\xi_{1}$, we get

$$
A\left[\rho\left(1-p^{*}\right)^{\gamma} q ; l, \xi\right] \leq 0
$$

Taking the super limit in the above inequality, we arrive at

$$
\limsup _{\xi \rightarrow \infty} A\left[\rho\left(1-p^{*}\right)^{\gamma} q ; l, \xi\right] \leq 0
$$

which is a contradiction to (31). This completes the proof of Theorem 3.2.

Remark 1 Look at inequality (21) from Theorem 3.1, we find that the function $R(\xi, h, l)=$ $\frac{\rho^{\prime}(\xi, h, l)}{\rho(\xi, h, l)}-\frac{\alpha_{3}(h)}{\alpha_{2}(h)}+2 \varphi(\xi, h, l)$ cannot judge the sign. And we note that the function $G(h)$ is affected by the damping function $g(\xi, c)$. Thus, we get a function $\rho(\xi, h, l)$ which can make function $R(\xi, h, l)=0$, so that $g(\xi, c)$ disappears in our criteria.

Theorem 3.3 Let $\left(A_{1}\right)-\left(A_{5}\right)$ and (13) hold. Assume that, for each $l \geq \xi_{0}$, there exists a positive function $\rho(\xi) \in C^{1}\left(\left[\xi_{0}, \infty\right), R^{+}\right)$satisfying $\rho^{\prime}(\xi)<0$ such that

$$
\limsup _{\xi \rightarrow \infty} \frac{\epsilon(\xi)}{\rho(\xi)}\left[\int_{g\left(\xi_{0}, c\right)}^{g(\xi, c)}\left(\frac{1}{\alpha_{1}(\varsigma)}\right)^{\frac{1}{\gamma}}\left(\int_{\xi_{0}}^{\varsigma} \frac{1}{\alpha_{2}(s)} d s\right)^{\frac{1}{\gamma}} d \varsigma\right]^{\gamma}>1
$$

where $\epsilon(\xi)=\int_{\xi}^{\xi^{\prime}} \rho(\varsigma)\left(1-p^{*}\right)^{\gamma} q(\varsigma) d \varsigma$ and $\xi^{\prime}>\xi$. Then every solution of Eq. (1) is oscillatory or converges to zero.

Proof Suppose the contrary, without loss of generality, we can assume that $x(\xi)$ is an eventually positive solution of Eq. (1) for $\xi>\xi_{0}$. Similar to the proof of Theorem 3.1, case 2 always hold and case 1 still satisfies (23), (26), (27), and (28) for $\xi>\xi_{1}$. By inequality (28), using $w(\xi)>0, \rho^{\prime}(\xi)<0$ and $\left(A_{1}\right)-\left(A_{5}\right)$, it is clear that $w^{\prime}(\xi)<0$. Multiplying both 
sides of inequality (26) with $g^{\prime}(\xi, c)$, then integrating this inequality from $\xi_{1}$ to $\xi$, note $\left(\alpha_{2}(\xi)\left(\alpha_{1}(\xi)\left(y^{\prime}(\xi)\right)^{\gamma}\right)^{\prime}\right)^{\prime}<0$ from (23), we have

$$
\begin{aligned}
& y(g(\xi, c)) \\
& \geq \int_{\xi_{1}}^{\xi}\left(\alpha_{2}(\varsigma)\left(\alpha_{1}(\varsigma)\left(y^{\prime}(\varsigma)\right)^{\gamma}\right)^{\prime}\right)^{\frac{1}{\gamma}} \frac{g^{\prime}(\varsigma, c)}{\alpha_{1}^{\frac{1}{\gamma}}(g(\varsigma, c))}\left(\int_{\xi_{1}}^{g(\varsigma, c)} \frac{1}{\alpha_{2}(s)} d s\right)^{\frac{1}{\gamma}} d \varsigma \\
& \geq\left(\alpha_{2}(\xi)\left(\alpha_{1}(\xi)\left(y^{\prime}(\xi)\right)^{\gamma}\right)^{\prime}\right)^{\frac{1}{\gamma}} \int_{\xi_{1}}^{\xi} \frac{g^{\prime}(\varsigma, c)}{\alpha_{1}^{\frac{1}{\gamma}}(g(\varsigma, c))}\left(\int_{\xi_{1}}^{g(\varsigma, c)} \frac{1}{\alpha_{2}(s)} d s\right)^{\frac{1}{\gamma}} d \varsigma \\
& =\left(\alpha_{2}(\xi)\left(\alpha_{1}(\xi)\left(y^{\prime}(\xi)\right)^{\gamma}\right)^{\prime}\right)^{\frac{1}{\gamma}} \int_{g\left(\xi_{1}, c\right)}^{g(\xi, c)}\left(\frac{1}{\alpha_{1}(\varsigma)}\right)^{\frac{1}{\gamma}}\left(\int_{\xi_{1}}^{\varsigma} \frac{1}{\alpha_{2}(s)} d s\right)^{\frac{1}{\gamma}} d \varsigma .
\end{aligned}
$$

From (28), note $\rho^{\prime}(\xi)<0$ and $w(\xi)>0$, we obtain

$$
w^{\prime}(\xi) \leq-\rho(\xi)\left(1-p^{*}\right)^{\gamma} q(\xi)
$$

Integrating the above inequality from $\xi$ to $\xi^{\prime}$, for $\xi<\xi^{\prime}$,

$$
\begin{aligned}
w(\xi) & \geq w\left(\xi^{\prime}\right)+\int_{\xi}^{\xi^{\prime}} \rho(\varsigma)\left(1-p^{*}\right)^{\gamma} q(\varsigma) d \varsigma \\
& \geq \int_{\xi}^{\xi^{\prime}} \rho(\varsigma)\left(1-p^{*}\right)^{\gamma} q(\varsigma) d \varsigma .
\end{aligned}
$$

And we assert

$$
\int_{\xi}^{\xi^{\prime}} \rho(\varsigma)\left(1-p^{*}\right)^{\gamma} q(\varsigma) d \varsigma<\infty
$$

Otherwise $w\left(\xi^{\prime}\right) \leq w(\xi)-\int_{\xi}^{\xi^{\prime}} \rho(\varsigma)\left(1-p^{*}\right)^{\gamma} q(\varsigma) d \varsigma \rightarrow-\infty$ for all $\xi^{\prime}>\xi$. From (27), we have that $w\left(\xi^{\prime}\right)>0$ is positive, which is a contradiction.

Next, from (27) and (33), we get

$$
\begin{aligned}
\frac{1}{w(\xi)} & =\frac{y^{\gamma}(g(\xi, c))}{\rho(\xi) \alpha_{2}(\xi)\left(\alpha_{1}(\xi)\left(y^{\prime}(\xi)\right)^{\gamma}\right)^{\prime}} \\
& \geq \frac{1}{\rho(\xi)}\left[\int_{g\left(\xi_{1}, c\right)}^{g(\xi, c)}\left(\frac{1}{\alpha_{1}(\varsigma)}\right)^{\frac{1}{\gamma}}\left(\int_{\xi_{1}}^{\varsigma} \frac{1}{\alpha_{2}(s)} d s\right)^{\frac{1}{\gamma}} d \varsigma\right]^{\gamma} .
\end{aligned}
$$

Thus it follows from (35) that

$$
\begin{aligned}
& \frac{w(\xi)}{\rho(\xi)}\left[\int_{g\left(\xi_{0}, c\right)}^{g(\xi, c)}\left(\frac{1}{\alpha_{1}(\varsigma)}\right)^{\frac{1}{\gamma}}\left(\int_{\xi_{0}}^{\varsigma} \frac{1}{\alpha_{2}(s)} d s\right)^{\frac{1}{\gamma}} d \varsigma\right]^{\gamma} \\
& \leq\left[\frac{\int_{g\left(\xi_{0}, c\right)}^{g(\xi, c)}\left(\frac{1}{\alpha_{1}(\varsigma)}\right)^{\frac{1}{\gamma}}\left(\int_{\xi_{0}}^{\varsigma} \frac{1}{\alpha_{2}(s)} d s\right)^{\frac{1}{\gamma}} d \varsigma}{\int_{g\left(\xi_{1}, c\right)}^{g(\xi, c)}\left(\frac{1}{\alpha_{1}(\varsigma)}\right)^{\frac{1}{\gamma}}\left(\int_{\xi_{1}}^{\varsigma} \frac{1}{\alpha_{2}(s)} d s\right)^{\frac{1}{\gamma}} d \varsigma}\right]^{\gamma} .
\end{aligned}
$$


Taking the super limit in the above inequality, we have

$$
\limsup _{\xi \rightarrow \infty} \frac{w(\xi)}{\rho(\xi)}\left[\int_{g\left(\xi_{0}, c\right)}^{g(\xi, c)}\left(\frac{1}{\alpha_{1}(\varsigma)}\right)^{\frac{1}{\gamma}}\left(\int_{\xi_{0}}^{\varsigma} \frac{1}{\alpha_{2}(s)} d s\right)^{\frac{1}{\gamma}} d \varsigma\right]^{\gamma} \leq 1 .
$$

Using (34) and (36), we can easily get a contradiction in (32). The proof is complete.

Theorem 3.4 Let $\left(A_{1}\right)-\left(A_{5}\right)$ and (13) hold. Suppose that $\left\{\vartheta_{n}(\xi)\right\}_{n=0}^{\infty}$ is a sequence of functions defined as

$$
\vartheta_{n}(\xi)=\int_{\xi}^{\varepsilon(\xi)} \widetilde{G}(s) \vartheta_{n-1}^{\frac{\gamma+1}{\gamma}}(s) d s+\vartheta_{0}(\xi), \quad \xi \geq \xi_{1}, n=1,2,3, \ldots
$$

where $\widetilde{G}(s)=\gamma \rho^{-\frac{1}{\gamma}}(s) G(s), \vartheta_{0}(\xi)=\int_{\xi}^{\varepsilon(\xi)} \rho(s)\left(1-p^{*}\right)^{\gamma} q(s) d s$ for $\xi>\xi_{0}$, and $\varepsilon(\xi) \in C^{1}\left(\left(\xi_{0}, \infty\right)\right.$, $(\xi, \infty)), \varepsilon^{\prime}(\xi)>0$. Assume that there exists a positive function $\rho(\xi) \in C^{1}\left(\left[\xi_{0}, \infty\right), R^{+}\right)$, satisfying $\rho^{\prime}(\xi)<0$ such that

$$
\limsup _{\xi \rightarrow \infty} \frac{\vartheta_{n}(\xi)}{\rho(\xi)}\left[\int_{g(\xi 0, c)}^{g(\xi, c)}\left(\frac{1}{\alpha_{1}(\varsigma)}\right)^{\frac{1}{\gamma}}\left(\int_{\xi_{0}}^{\varsigma} \frac{1}{\alpha_{2}(s)} d s\right)^{\frac{1}{\gamma}} d \varsigma\right]^{\gamma}>1
$$

Then every solution of Eq. (1) is oscillatory or converges to zero.

Proof Assume that $x(\xi)$ is a positive solution of Eq. (1), then similar to the proof of Theorem 3.1 and Theorem 3.3, the case 2 hold, and we deduce that (27), (28), (36) in case 1 hold. Next, we prove that there exists a positive $\vartheta(\xi)$ on $\left[\xi_{1}, \infty\right)$ such that $\lim _{\xi \rightarrow \infty} \vartheta_{n}(\xi)=\vartheta(\xi)$, $\vartheta(\xi)=\int_{\xi}^{\varepsilon(\xi)} \widetilde{G}(s) \vartheta^{\frac{\gamma+1}{\gamma}}(s) d s+\vartheta_{0}(\xi)$, and $w(\xi) \geq \vartheta_{n}(\xi)$.

In fact from $(28), \rho^{\prime}(\xi)<0$, and $\widetilde{G}(\xi)=\gamma \rho^{-\frac{1}{\gamma}}(\xi) G(\xi)$, we get

$$
w^{\prime}(\xi)+\widetilde{G}(\xi) w^{\frac{\gamma+1}{\gamma}}(\xi)+\rho(\xi)\left(1-p^{*}\right)^{\gamma} q(\xi) \leq 0 .
$$

Then integrating (39) from $\xi$ to $\varepsilon(\xi)$ and setting $\vartheta_{0}(\xi)=\int_{\xi}^{\varepsilon(\xi)} \rho(s)\left(1-p^{*}\right)^{\gamma} q(s) d s$, we have

$$
w(\varepsilon(\xi))-w(\xi)+\int_{\xi}^{\varepsilon(\xi)} \widetilde{G}(s) w^{\frac{\gamma+1}{\gamma}}(s) d s+\vartheta_{0}(\xi) \leq 0 .
$$

From $\left(A_{2}\right),\left(A_{3}\right)$ and $\rho(\xi) \in C^{1}\left(\left[\xi_{1}, \infty\right), R^{+}\right)$, we get $\vartheta_{0}(\xi)>0$ and

$$
w(\varepsilon(\xi))-w(\xi)+\int_{\xi}^{\varepsilon(\xi)} \widetilde{G}(s) w^{\frac{\gamma+1}{\gamma}}(s) d s \leq 0 .
$$

Then we conclude that

$$
\int_{\xi}^{\varepsilon(\xi)} \widetilde{G}(s) w^{\frac{\gamma+1}{\gamma}}(s) d s<\infty
$$

otherwise, $w(\varepsilon(\xi)) \leq w(\xi)-\int_{\xi}^{\varepsilon(\xi)} \widetilde{G}(s) w^{\frac{\gamma+1}{\gamma}}(s) d \varsigma \rightarrow-\infty$ for all $\xi>\xi_{1}$. From (27), we have that $w(\varepsilon(\xi))$ is positive, which is a contradiction. Similarly, we also have $\vartheta_{0}(\xi)<\infty$. We 
observe that function $\widetilde{G}(s)>0$, from (28), we have that $w(\xi)$ is positive and decreasing. By (40), we get

$$
w(\xi) \geq \int_{\xi}^{\varepsilon(\xi)} \widetilde{G}(s) w^{\frac{\gamma+1}{\gamma}}(s) d s+\vartheta_{0}(\xi)
$$

That means $w(\xi) \geq \vartheta_{0}(\xi)$. In addition, from $\widetilde{G}(\xi)>0$, we have $\vartheta_{n}(\xi) \leq \vartheta_{n+1}(\xi)$ for $\xi>\xi_{1}$ always holds. By $w(\xi) \geq \vartheta_{0}(\xi)$ and (43), we have

$$
w(\xi) \geq \int_{\xi}^{\varepsilon(\xi)} \widetilde{G}(s) \vartheta_{0}^{\frac{\gamma+1}{\gamma}}(s) d s+\vartheta_{0}(\xi)=\vartheta_{1}(\xi)
$$

Then we have

$$
w(\xi) \geq \int_{\xi}^{\varepsilon(\xi)} \widetilde{G}(s) \vartheta_{1}^{\frac{\gamma+1}{\gamma}}(s) d s+\vartheta_{0}(\xi)=\vartheta_{2}(\xi)
$$

Inductively, we obtain $w(\xi) \geq \vartheta_{n}(\xi), n=1,2,3, \ldots$, for $\xi>\xi_{1}$.

Since the sequence $\left\{\vartheta_{n}(\xi)\right\}_{n=0}^{\infty}$ is increasing and bounded, it converges to $\vartheta(\xi)$. Then, using Lebesgue's monotone convergence theorem and putting $n \rightarrow \infty$ in (37), we get $\vartheta(\xi)=\int_{\xi}^{\varepsilon(\xi)} \widetilde{G}(s) \vartheta^{\frac{\gamma+1}{\gamma}}(s) d s+\vartheta_{0}(\xi)$. Then, from $(36)$ and $w(\xi) \geq \vartheta_{n}(\xi)$, we also get a contradiction to (38). The proof is complete.

Remark 2 We give a Riccati-type function satisfying $\rho^{\prime}(\xi)<0$ such that the function $w(\xi)$ is bounded. If we change the condition of $\rho^{\prime}(\xi)<0$ to $\frac{\rho^{\prime}(\xi)}{\rho(\xi)}-\frac{\alpha_{3}(\xi)}{\alpha_{2}(\xi)}<0$ in Theorem 3.3 or Theorem 3.4, we also have that the function $w(\xi)$ is bounded. Then, by the same proof, we get new oscillation criteria.

\section{Example}

Consider the following differential equation:

$$
\begin{aligned}
\left(\frac{1}{\xi}\left(\xi\left(\left(x(\xi)+\int_{0}^{1} \frac{1}{2} x\left(\frac{\xi}{2}\right) d \sigma\right)\right)^{3}\right)^{3}\right)^{\prime} \\
\quad+\frac{1}{\xi^{2}}\left(\xi\left(\left(x(\xi)+\int_{0}^{1} \frac{1}{2} x\left(\frac{\xi}{2}\right) d \sigma\right)^{\prime}\right)^{3}\right)^{\prime}+\int_{0}^{1} \lambda x\left(\frac{\xi}{e}\right) d s=0,
\end{aligned}
$$

for $\xi \in(0, \infty)$.

Let $\alpha_{1}(\xi)=\xi, \alpha_{2}(\xi)=\frac{1}{\xi}, \alpha_{3}(\xi)=\frac{1}{\xi^{2}}, p^{*}=\frac{1}{2}, \tau(\xi, \sigma)=\frac{\xi}{2}, F(\xi, s, x)=\lambda x, g(\xi, s)=\frac{\xi}{e}, \gamma=3$, $a=0, b=1, c=0, d=1, \eta(\xi)=\xi$ in Eq. (1). Then we have $q(\xi, s)=\lambda$, and we also obtain $q(\xi)=\lambda, \int_{\xi_{0}}^{\infty} \alpha_{1}(\xi)^{-\frac{1}{\gamma}} d \xi=\infty, \int_{\xi_{0}}^{\infty} \frac{1}{\alpha_{2}(\xi)} \exp \left(-\int_{\xi_{0}}^{\xi} \frac{\alpha_{3}(s)}{\alpha_{2}(s)} d s\right) d \xi=\infty$. And (13) holds for $\lambda>0$.

We give $\Phi(\xi, h, l)=(\xi-h)^{\alpha}(h-l)^{\beta}, \alpha>\frac{1}{2}, \beta>\frac{1}{2}$. From (4), we get $\varphi(\xi, h, l)=\frac{\beta \xi-(\alpha+\beta) h+\alpha l}{(\xi-h)(h-l)}$, by simple computation, we have

$$
\begin{gathered}
\exp \left(\int_{\xi_{0}}^{h}\left(\frac{\alpha_{3}(s)}{\alpha_{2}(s)}-\frac{2(\beta \xi-(\alpha+\beta) s+\alpha l)}{(\xi-s)(s-l)}\right) d s\right) \\
=\exp \left(\int_{\xi_{0}}^{h}\left(\frac{1}{s}-2\left(\frac{-\alpha}{\xi-s}+\frac{\beta}{s-l}\right)\right) d s\right)
\end{gathered}
$$




$$
\begin{aligned}
& =\exp \left(\int_{\xi_{0}}^{h} \frac{1}{s} d s\right) \cdot \exp \left(2 \alpha \int_{\xi_{0}}^{h} \frac{1}{\xi-s} d s\right) \cdot \exp \left(-2 \beta \int_{\xi_{0}}^{h} \frac{1}{s-l} d s\right) \\
& =\frac{h}{\xi_{0}} \cdot\left(\frac{\xi-h}{\xi-\xi_{0}}\right)^{-2 \alpha} \cdot\left(\frac{h-l}{\xi_{0}-l}\right)^{-2 \beta}=\frac{h(\xi-h)^{-2 \alpha}(h-l)^{-2 \beta}}{\xi_{0}\left(\xi-\xi_{0}\right)^{-2 \alpha}\left(\xi_{0}-l\right)^{-2 \beta}} .
\end{aligned}
$$

Then we have

$$
\begin{aligned}
& \limsup _{\xi \rightarrow \infty} \int_{l}^{\xi}(\xi-h)^{2 \alpha}(h-l)^{2 \beta} \rho(\xi, h, l)\left(1-p^{*}\right)^{\gamma} q(h) d h \\
& =\limsup _{\xi \rightarrow \infty} \frac{\lambda\left(\xi-\xi_{0}\right)^{2 \alpha}}{8 \xi_{0}\left(\xi_{0}-l\right)^{-2 \beta}} \int_{l}^{\xi}(\xi-h)^{2 \alpha}(h-l)^{2 \beta} h(\xi-h)^{-2 \alpha}(h-l)^{-2 \beta} d h \\
& =\limsup _{\xi \rightarrow \infty} \frac{\lambda\left(\xi^{2}-l^{2}\right)\left(\xi-\xi_{0}\right)^{2 \alpha}}{16 \xi_{0}\left(\xi_{0}-l\right)^{-2 \beta}} \text {. }
\end{aligned}
$$

Because of $\limsup _{\xi \rightarrow \infty} \frac{\left(\xi^{2}-l^{2}\right)\left(\xi-\xi_{0}\right)^{2 \alpha}}{16 \xi_{0}\left(\xi_{0}-l\right)^{-2 \beta}} \rightarrow+\infty$, we get that (47) is positive for $\lambda>0$. Using Theorem 3.2 and operator $A[\cdot ; l, \xi]$, we have that the solution of (46) is oscillatory or converges to zero for $\lambda>0$.

\section{Conclusion}

In this paper, we study the oscillation criteria of TOND differential equations with distributed deviating arguments and damping. Under the condition of $\left(A_{1}\right)$, we analyze the distribution function $y(\xi)$ which is related to the solution. Then we give some sufficient condition which ensures that every solution of Eq. (1) is oscillatory or converges to zero. In future work, we would like to get some other oscillation criteria of Eq. (1) when $\int_{a}^{b} p(\xi, \sigma) d \sigma>1$ in $\left(A_{2}\right)$. In fact, the case of "> 1" has been involved in studies of secondorder and even-order neutral differential equations. See, e.g., the papers [24-27] for more details.

Acknowledgements

The authors are grateful to the reviewers for their valuable and insightful comments.

Funding

This research is supported by the National Natural Science Foundation of China (No. 11671227) and the Natural Science Foundation of Shandong Province (No. ZR2019MA034).

Availability of data and materials Not applicable.

\section{Declarations}

Competing interests

The authors declare that they have no competing interests.

Authors' contributions

All authors read and approved the final manuscript.

\section{Author details}

${ }^{1}$ School of Mathematical Sciences, Qufu Normal University, Shandong, P.R. China. ${ }^{2}$ Army Academy of Armored Forces, Beijing, P.R. China.

\section{Publisher's Note}

Springer Nature remains neutral with regard to jurisdictional claims in published maps and institutional affiliations. 


\section{References}

1. Arino, O., Hbid, M.L., Dads, E.A.: Oscillation Theory for Difference and Functional Differential Equations. Springer, Berlin (2006)

2. Ladde, G.S., Zhang, B.G., Lakshmikantham, V.: Oscillation Theory of Differential Equations with Deviating Arguments. Dekker, New York (1987)

3. Chatzarakis, G.E., Grace, S.R., Jadlovska, I., Li, T., Tunc, E.: Oscillation criteria for third-order Emden-Fowler differential equations with unbounded neutral coefficients. Complexity 2019, Article ID 5691758 (2019)

4. Bohner, M., Hassan, T.S., Li, T.: Fite-Hille-Wintner-type oscillation criteria for second-order half-linear dynamic equations with deviating arguments. Indag. Math. 29, 548-560 (2018)

5. Dzurina, J., Grace, S.R., Jadlovska, I., Li, T.: Oscillation criteria for second-order Emden-Fowler delay differential equations with a sublinear neutral term. Math. Nachr. 293, 910-922 (2020)

6. Li, T., Pintus, N., Viglialoro, G.: Properties of solutions to porous medium problems with different sources and boundary conditions. Z. Angew. Math. Phys. 70, 1-18 (2019)

7. Li, T., Viglialoro, G.: Boundedness for a nonlocal reaction chemotaxis model even in the attraction-dominated regime. Differ. Integral Equ. 34, 315-366 (2021)

8. Zhang, C., Agarwal, R.P., Li, T.: Oscillation and asymptotic behavior of higher-order delay differential equations with p-Laplacian like operators. J. Math. Anal. Appl. 409, 1093-1106 (2014)

9. Wei, M., Jiang, C., Li, T.: Oscillation of third-order neutral differential equations with damping and distributed delay. Adv. Differ. Equ. 2019, 426 (2019)

10. Wei, M.H., Zhang, M.L., Liu, X.L., Yu, Y.H.: Oscillation criteria for a class of third order neutral distributed delay differential equations with damping. J. Math. Comput. Sci. 2019, 19-28 (2019)

11. Bohner, M., Grace, S.R., Sager, I., Tunc, E.: Oscillation of third-order nonlinear damped delay differential equations. Appl. Math. Comput. 278, 21-32 (2016)

12. Shi, Y., Han, Z., Hou, C.: Oscillation criteria for third order neutral Emden-Fowler delay dynamic equations on time scales. Appl. Math. Comput. 55, 175-190 (2017)

13. Sui, Y., Han, Z:: Oscillation of third-order nonlinear delay dynamic equation with damping term on time scales. J. Appl. Math. Comput. 58, 577-599(2018)

14. Jiang, C., Jiang, Y., Li, T.: Asymptotic behavior of third-order differential equations with nonpositive neutral coefficients and distributed deviating arguments. Adv. Differ. Equ. 2016, 105 (2016)

15. Li, T., Rogovchenko, Y.V.: On the asymptotic behavior of solutions to a class of third-order nonlinear neutral differential equations. Appl. Math. Lett. 105, 106293 (2020)

16. Tian, Y., Cai, Y., Fu, Y., Li, T.: Oscillation and asymptotic behavior of third-order neutral differential equations with distributed deviating arguments. Adv. Differ. Equ. 2015, 267 (2015)

17. Xu, R., Meng, F.: New Kamenev-type oscillation criteria for second order neutral nonlinear differential equations. Appl. Math. Comput. 118, 1364-1370 (2007)

18. Liu, L., Bai, Y.: New oscillation criteria for second-order nonlinear neutral delay differential equations. J. Comput. Appl. Math. 231, 657-663 (2009)

19. Zhao, J., Meng, F.: Oscillation criteria for second-order neutral equations with distributed deviating argument. Appl. Math. Comput. 206, 485-493 (2008)

20. Jiang, C., Tian, Y., Jiang, Y., Li, T.: Some oscillation results for nonlinear second-order differential equations with damping. Adv. Differ. Equ. 2015, 354 (2015)

21. Zhang, M., Chen, W., Elsheikh, M., Sallam, R.A., Hassan, A.M., Li, T.: Oscillation criteria for second-order nonlinear delay dynamic equations of neutral type. Adv. Differ. Equ. 2018, 26 (2018)

22. Candan, T:: Oscillatory behavior of second order nonlinear neutral differential equations with distributed deviating arguments. Appl. Math. Comput. 262, 199-203 (2015)

23. Meng, F., Xu, R.: Oscillation criteria for certain even order quasi-linear neutral differential equations with deviating arguments. Appl. Math. Comput. 190, 458-464 (2007)

24. Li, T., Rogovchenko, Y.V.: Oscillation of second-order neutral differential equations. Math. Nachr. 288, 1150-1162 (2015)

25. Agarwal, R.P., Bohner, M., Li, T., Zhang, C.: A new approach in the study of oscillatory behavior of even-order neutral delay differential equations. Appl. Math. Comput. 225, 787-794 (2013)

26. Li, T., Rogovchenko, Y.V.: Oscillation criteria for even-order neutral differential equations. Appl. Math. Lett. 61, 35-41 (2016)

27. Li, T., Rogovchenko, Y.V.: Oscillation criteria for second-order superlinear Emden-Fowler neutral differential equations Monatshefte Math. 184, 489-500 (2017) 